\title{
Simulación de la influencia de las demoras de autobuses en la operación de las paradas de bus de Riobamba
}

Simulation of the influence of bus delays in the operation of the Riobamba

bus stops

1 Germán Ulises Moreno Arias

https://orcid.org/0000-0002-9616-6616 Escuela Superior Politécnica de Chimborazo (ESPOCH), Carrera de Gestión de Transporte, Chimborazo, Riobamba, Ecuador ulises.moreno@espoch.edu.ec

2 Arquímedes Xavier Haro Velastegui

https://orcid.org/0000-0003-3391-5082 Escuela Superior Politécnica de Chimborazo (ESPOCH), Carrera de Gestión de Transporte, Chimborazo, Riobamba, Ecuador aharo@espoch.edu.ec

Artículo de Investigación Científica y Tecnológica

Enviado: 11/12/2021

Revisado: $26 / 12 / 2021$

Aceptado: 18/01/2022

Publicado:07/02/2022

DOI: https://doi.org/10.33262/ap.v4i1.145

demoras de autobuses en la operación de las paradas de bus de Riobamba.

Cítese: $\quad$ AlfaPublicaciones, 4(1), 97-116. https://doi.org/10.33262/ap.v4i1.145

ALFA PUBLICACIONES, es una Revista Multidisciplinar, Trimestral, que se publicará en soporte electrónico tiene como misión contribuir a la formación de profesionales competentes con visión humanística y crítica que sean capaces de exponer sus resultados investigativos y científicos en la misma medida que se promueva mediante su intervención cambios positivos en la sociedad. https://alfapublicaciones.com

Ciencia La revista es editada por la Editorial Ciencia Digital (Editorial de prestigio registrada en la Cámara Ecuatoriana de Libro con No de Afiliación 663) www.celibro.org.ec 
Palabras

claves:

simulación, sistemas, demoras, entidades, locaciones.

Keywords: simulation, systems, delays, entities, locations

\section{Resumen}

Introducción. El transporte público juega un papel importante en la mayoría de las áreas pobladas. Especialmente en las regiones urbanas, estos sistemas de transporte son ampliamente utilizados. Pero desafortunadamente, este tipo de servicio es a menudo objeto de quejas. Los clientes están molestos por los "precios injustos", por el "mal servicio" y, en particular, se molestan en caso de retrasos. Respecto a lo particular, este artículo presenta un modelo de simulación por computadora desarrollado con el Software Promodel. Objetivo. El propósito es, evaluar mediante la técnica de simulación cómo influyen las demoras de los autobuses entre paradas, por dispositivos para el control de tránsito e indisciplinas de los conductores en la operación de las paradas de bus de la ciudad de Riobamba. Metodología. En el ámbito metodológico de la simulación se procede a desarrollar un modelo base. Antes de diseñar este modelo concretamos el objetivo de investigación en la forma de salidas numéricas específicas; estas son, los promedios de pasajeros por bus, en todo el sistema de ruta de la línea 2, por cada grupo de demoras contempladas en la hipótesis de investigación, como también, los tiempos promedios de espera de los pasajeros (véase Tablas 1 y 2). Resultados. El modelo permite obtener resultados simulados si del escenario del sistema completo anulamos, por ejemplo, las demoras por indisciplinas (Dpi) para obtener un nuevo escenario: Dep+Dpdis. Ahora tenemos una nueva versión del modelo que con el soporte de ProModel nos permite observar las salidas numéricas bajo la influencia del par: Dep+Dpdis; y así, con los demás. Conclusión. El modelo de simulación por computadora permite concluir que el promedio de pasajeros y su tiempo de espera en las paradas de todo el sistema no se ven afectados por cada tipo de demora que experimentan los autobuses.

\section{Abstract}

Introduction. Public transportation plays an important role in most populated areas. Especially in urban regions, these transportation systems are widely used. But unfortunately, this type of service is often the subject of complaints. Customers are annoyed by "unfair prices", by "bad service" and, in particular, they are annoyed in case of delays. Regarding the particular, this article presents a computer simulation model developed with the Promodel Software. 
Objective. The purpose is to evaluate, through the simulation technique, how the delays of the buses between stops influence the traffic control devices and indiscipline of the drivers in the operation of the bus stops in the city of Riobamba. Methodology. In the methodological field of simulation, a base model is developed. Before designing this model, we specified the research objective in the form of specific numerical outputs; These are the average number of passengers per bus, throughout the line 2 route system, for each group of delays contemplated in the research hypothesis, as well as the average passenger waiting times (see Tables 1 and 2). Results. The model allows simulated results to be obtained if from the complete system scenario we cancel, for example, the delays due to indiscipline (Dpi) to obtain a new scenario: Dep+Dpdis. Now we have a new version of the model that with the support of ProModel allows us to observe the numerical outputs under the influence of the pair: Dep+Dpdis; and so, with the others. Conclusion. The computer simulation model allows us to conclude that the average number of passengers and their waiting time at stops throughout the system are not affected by each type of delay experienced by buses.

\section{Introducción}

El estudio de la movilidad urbana implica una etapa de toma de información, destinada a la caracterización tanto de la oferta como de la demanda de transporte (Ortúzar, 2015). Este documento hace énfasis en lo segundo al "evaluar mediante un modelo de simulación por computadora cómo influyen las demoras de los autobuses entre paradas, por dispositivos para el control de tránsito e indisciplinas de los conductores en la operación de las paradas de bus de la ciudad de Riobamba, Provincia de Chimborazo-Ecuador".

Aunque las técnicas de planificación de transporte han ido experimentando fuertes cambios en las últimas décadas, los problemas de transporte, particularmente en el caso urbano, no han variado gran cosa a nivel local (Ortúzar, 2015). Así, cada vez son más las quejas de las personas que utilizan el servicio de autobuses de la línea 2 en la ciudad de Riobamba. Estos clientes de las horas de mayor afluencia están cansados de esperar el autobús por la mañana. Más aun, mientras más demoran éstos, más se llenan las paradas. A medida que avanza el invierno o el verano, los usuarios se incomodan cada vez más al no haber paradas cubiertas donde tomar asiento. 
Este trabajo presenta una metodología basada en la modelación y simulación por computadora, que permite abordar la problemática antes mencionada. La complejidad en la operación de este tipo de sistemas requiere de una modelación cada vez más apegada a la realidad. Las estimaciones generales del modelo del rendimiento del sistema (tiempos de espera y colas) se comparan con las mediciones de campo de los tiempos de espera y de colas. Se realizan ajustes finos para permitir que el modelo coincida mejor con las mediciones de campo (Barceló, 2010). El modelo desarrollado con apoyo del Software ProModel (García et al., 2013), se fundamenta en la teoría de colas. Cada locación o parada de bus se reduce a la interacción simultánea de dos entidades, a saber: bus y pasajero.

Para el estudio de sistemas modelados mediante modelos de colas cuya solución analítica se desconoce, o bien es muy difícil de obtener, se suele recurrir a técnicas de simulación que, mediante el empleo de programas de computación, permiten obtener estimaciones de los parámetros de interés del comportamiento del sistema (Pazos et al., 2003).

En el campo de la investigación que nos concierne, la formación de colas de tráfico durante los períodos de congestionamiento es una fuente de demoras considerables y da como resultado una pérdida del rendimiento de las vías. En condiciones extremas, la demora en las colas puede representar el $90 \%$ o más del tiempo total de viaje del conductor. Dado esto, es esencial en el análisis de demoras y tiempos de espera desarrollar una comprensión clara de las características de la formación y disipación de las colas junto con formulaciones matemáticas que puedan predecir los elementos relacionados con las colas.

\section{Metodología}

\section{Linea Piloto}

Se empieza realizando una breve descripción de la línea de buses. En este contexto, la línea 2 conocida con el nombre: 24 de mayo-Terminal- Bellavista, inicia su operación con un horario de 06h10 hasta las 22h30. Los vehículos que laboran diariamente sólo en esta línea; suman en total doce; de ahí que, en conjunto las líneas 1,2 y 3 trabajen con un intervalo de 2 y 3 minutos en horas pico y horas valle respectivamente. Por su parte, la frecuencia de despacho de las unidades de transporte de la línea 2 está regulada a intervalos de 8 minutos.

La ruta de la línea 2 (véase figura 1) de transporte público que se utiliza para la asignación de viajes de los autobuses, tiene una longitud de $26.8 \mathrm{Km}$. Además, se constituye por las principales vialidades de la ciudad, así como de sus intersecciones. 
Tiempos de viaje y velocidad

Otro aspecto importante que ayuda a estudiar y resolver el problema planteado, es establecer el tiempo de viaje y velocidad promedio. Es así que, El Consejo de Tránsito de Chimborazo en períodos pico mediante el método del vehículo flotante, que consiste en conducir un vehículo liviano tratando de mantener una velocidad representativa del flujo total, de acuerdo a las reglas de conducción, registra que en promedio el tiempo de recorrido para cubrir todo el trayecto es de 1hora, 10minutos.

Por otra parte, los estudios de las velocidades de marcha realizados por El Departamento de Ingeniería de Tránsito de la Policía de Chimborazo a lo largo de la ruta considerada de acuerdo con la técnica del vehículo medio, que consiste, en que el vehículo viaja de acuerdo a la apreciación que tenga el conductor de la velocidad predominante en el flujo de tránsito, determinan diversas velocidades de marcha, donde el promedio de las mismas se emplea como la velocidad de recorrido en el modelo, esto es: $20.586 \mathrm{~km} / \mathrm{h}$, o su equivalente registrada en la ventana entities de ProModel: $343.10 \mathrm{mpm}$.

\section{Figura 1}

Esquema general del servicio de transporte colectivo urbano "STCU" de la línea 2.

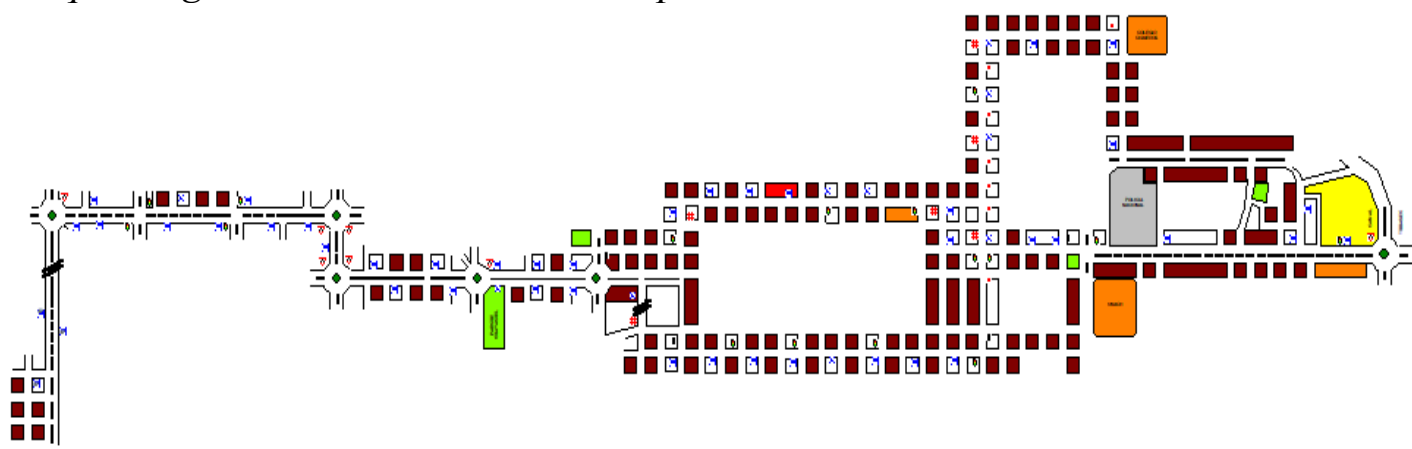

Nota: Esquema realizado en Software ProModel del servicio de transporte colectivo urbano de la ciudad de Riobamba de la ruta de la línea 2. Fuente: German Moreno

\section{Capacidad y demoras entre paradas}

También en el proceso de modelado es necesario contar con estas dos medidas; luego, es preciso tener en cuenta que estos conceptos se aplican a autobuses y paradas en vías urbanas para análisis, tanto de diseño como de operación. La capacidad depende de las unidades de servicio. La razón principal de su elección, es la necesidad de conocer en promedio el número de pasajeros que transportan, tanto de pies como sentados. Otra razón por la que se vincula al modelo de simulación es que, la cantidad de pasajeros en cola que se suben al vehículo (pasajeros servidos) depende de la capacidad legal permitida. En este contexto, El Departamento de Ingeniería de Tránsito de Chimborazo establece un intervalo de pasajeros sentados; a saber: 38-40; mientras tanto que la ley, en el caso de pasajeros parados, permite un $20 \%$ de la capacidad del autobús. 
Para los especialistas de transporte, las medidas velocidad, tiempos de viaje, demoras, tiempos entre paradas, etc. son de importancia, de ahí que, el informe técnico del Consejo Provincial de Tránsito establece un estimado entre paradas de 1 minuto \pm 0.2 segundos. Estos datos registrados en los dos párrafos se utilizan para el ajuste del modelo matemático de simulación.

\section{Tiempos de demoras entre paradas (DEP)}

La influencia de todas las demoras se mide como la diferencia, entre el tiempo que demora el bus entre una parada y la siguiente y las demoras producidas por los dispositivos para el control del tránsito más las provocadas por indisciplinas del conductor en las paradas. Entonces:

DEP = hora actual_parada $\mathbf{n}$ - hora de salida_parada $\mathbf{n}-\mathbf{1}+$ tiempo por indisciplinas

Como línea de programación en la ventana processing de ProModel, tenemos:

$$
\mathrm{DEP}=\text { CLOCK } 0 \text { - Hora_de_Entrada_Cola } \mathbf{n}+\mathbf{v a r} \text { _indisciplinas }
$$

Los tiempos por dispositivos para el control del tránsito se encuentran incluidos por declaración de variables y sus respectivas distribuciones de probabilidad, en la expresión:

\section{CLOCK () - Hora_de_Entrada_Cola $\mathbf{n}$}

Tiempos de demoras por dispositivos para el control del tránsito

Otra acción necesaria en el desarrollo del modelo de simulación por computadora es programar los tiempos de demoras de los autobuses cuando estos se detienen total o parcialmente frente a cada señal de Ceda el Paso, Pare o Semáforo. En el tiempo total de recorrido están incluidas todas aquellas demoras operacionales provocadas por los dispositivos de control. No incluye aquellas demoras fuera de la vía, como pueden ser las correspondientes a detenciones en gasolineras (Cárdenas, 2018).

Enseguida se ilustra con un ejemplo (véase figura 2) la distribución de probabilidad que mejor se ajusta a los datos de tiempos de demoras en el semáforo $\mathrm{N}^{\mathrm{0}} 1$, dirección NorteSur. 


\section{ISSN: 2773-7330}

\section{Figura 2}

Tiempos de demoras en el semáforo No 1

Normal (11.68,5.19) segundos

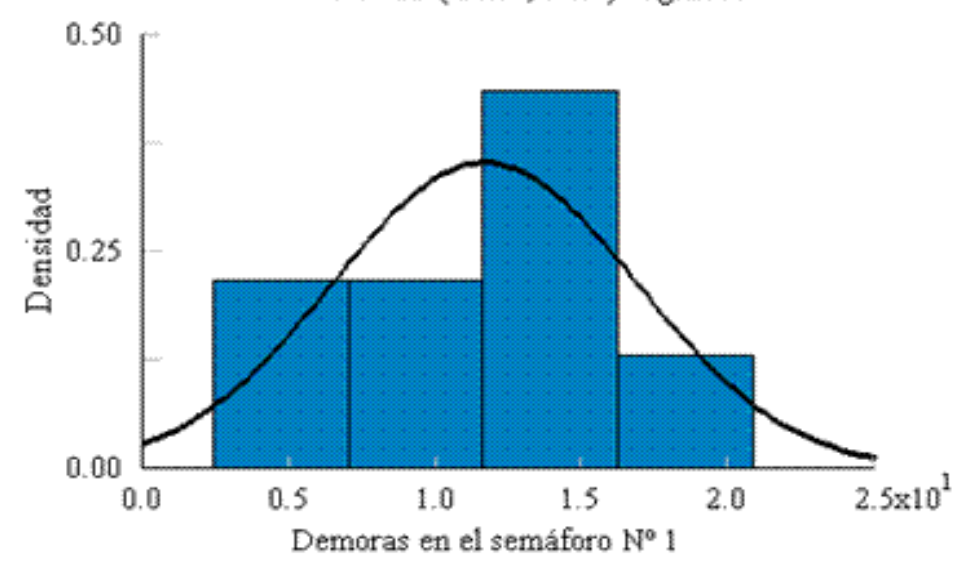

Nota: Distribución teórica obtenida mediante el Software StaFit que modela los datos recolectados en el primer semáforo de la ruta, en dirección Norte - Sur. Fuente: German Moreno

Una variable aleatoria continua $\mathrm{X}$ que tiene la distribución en forma de campana (figura 2) se denomina variable aleatoria normal. La fórmula matemática para la distribución de probabilidad de la variable normal depende de los dos parámetros $\mu$ y $\sigma$, su media y su desviación estándar, respectivamente (Walpole et al., 2012).

Tiempos de demoras por indisciplinas del conductor

En este apartado se consideran las demoras por indisciplinas del conductor (DPI); es decir, por demoras no planificadas en las paradas. El proceso que se realiza es medir la diferencia entre el tiempo total que demora el bus en una parada (tiempo de servicio "DTS") y el tiempo planificado de demoras por paradas que, según el Consejo Provincial de Tránsito al considerar el sistema integral de la línea 2, éste, por sus características y funciones debe operar en las paradas con una cantidad de tiempo de demora distribuido uniformemente entre 1 y 2 minutos, o sea: $U(0.5,1.5)$.

$$
=\mathbf{D T S}-\mathbf{U}(\mathbf{0 . 5}, \mathbf{1 . 5})
$$

\section{DPI}

Una distribución uniforme tiene la probabilidad de ocurrencias de un valor y es igualmente posible de ocurrir en cualquier lugar entre el menor valor $a$ y el mayor valor $b$ (Levine, et al., 2006).

\section{Modelo de colas}

En los modelos de colas existentes, las tareas producidas por los usuarios de una población llegan al sistema en un instante de tiempo aleatorio. Estas tareas demandan una 
cantidad de tiempo de servicio también aleatorio, y se sirven una a una o por grupos (Pazos et al., 2003). En las primeras paradas de bus la inclusión de los tiempos aleatorios de servicio da lugar al conteo de la variable cantidad de pasajeros servidos CPS. En las paradas donde se producen ascensos y descensos de pasajeros este tiempo de servicio da lugar a contar la cantidad de pasajeros servidos, así como, la cantidad de pasajeros que descienden del autobús CPDsc. Finalmente, después de completar el circuito, en las últimas paradas, el tiempo de descenso equivalente al tiempo de servicio da lugar al conteo del número de pasajeros que descienden del bus.

\section{Figura 3}

\section{Disciplina de cola por parada}

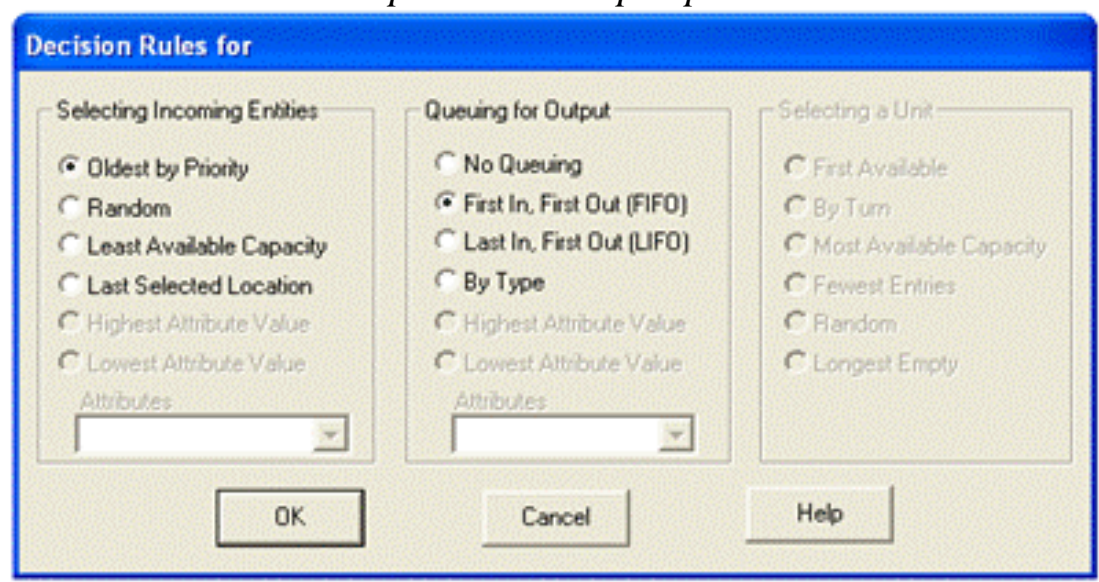

Nota: Ventana del Software ProModel que permite establecer la disciplina de cola en las paradas de buses. Fuente: German Moreno

El orden en el que se sirven los usuarios, a saber, en: QUsr_Paradan y QUsr_Descenso_Paradan, lo determina una disciplina de gestión de cola de la forma FIFO en ProModel. La figura 3 que se muestra define la disciplina adoptada en los cincuenta y cuatro modelos que componen el sistema (International Journal os Scientific Research in Science and Technology, 2018).

\section{Proceso de tiempo entre llegadas}

Otro parámetro que incide en el modelado son los tiempos entre llegadas de los usuarios del servicio de transporte colectivo urbano a las paradas. Este es un proceso estocástico continuo de tiempo (índice) discreto $\gamma$ (Pazos et al., 2003).

$$
\gamma \triangleq\left\{\gamma_{i} \triangleq T_{i+1-} T_{i} ; i=1,2,3, \ldots\right\}
$$

Obviamente, una vez caracterizado uno de estos tiempos, queda muy bien caracterizado el otro. La distribución exponencial con tasa $\lambda$, se muestra en la Figura 4. Esta aparece con frecuencia en muchas aplicaciones, incluidos los modelos de colas y la teoría de la confiabilidad (Kobayashi et al., 2012). Los tiempos entre llegadas de los pasajeros a la 
parada $\mathrm{N}^{0} 5$ se modelan con esta distribución obtenidas a través del programa Stat:Fit con un nivel de confianza del 95\%. Estos tiempos están dados en minutos y está basado en medición directa realizada por aforadores. El resto de ajustes de las demás paradas de bus se encuentran registrados en el propio modelo de simulación.

Figura 4

Distribución teórica: tiempos entre llegadas de pasajeros a la parada No 5

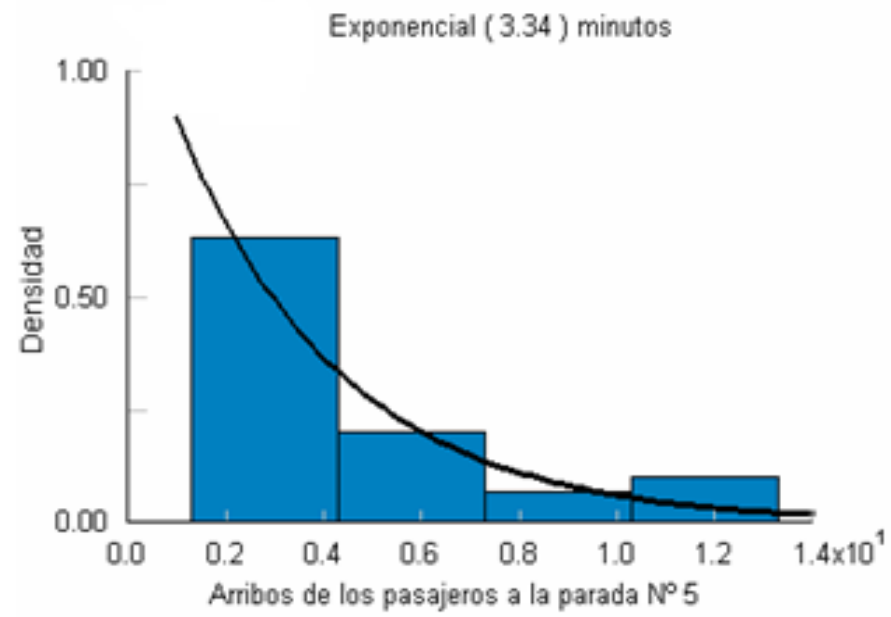

Nota: Distribución teórica obtenida mediante el Software StaFit que modela los datos recolectados de los tiempos entre llegadas de los pasajeros a la parada 5 dirección Norte - Sur. Fuente: German Moreno

Cantidad de pasajeros en la parada: CPP

El incremento de esta variable depende de los tiempos de servicio y de las demoras producidas en el sistema. Los contadores de la variable cantidad de pasajeros se colocan en el Layout y están identificados con el letrero CPP. En el programa de la figura 7, se muestra, la condición que debe cumplirse para que en las paradas de bus se produzca el incremento de pasajeros. identificados con el letrero CPP.

\section{Figura 5}

\section{Incremento de pasajeros en la Paradan}

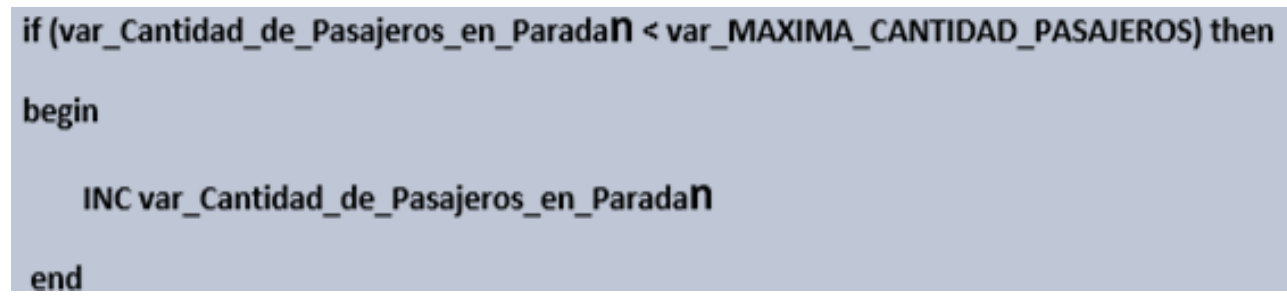

Nota: Línea de código que calcula el incremento de cantidad de pasajeros en la n-ésima parada de la ruta de la línea 2. Fuente: German Moreno 
En la publicación de la Organización de Planificación Metropolitana del Área Urbana de Memphis (MPO), se estableció que las paradas de autobús mal diseñadas pueden disminuir la satisfacción del cliente, hacer que el transporte público sea menos atractivo para los nuevos clientes potenciales y, potencialmente, hacer que la espera en las paradas sea insegura para los pasajeros.

\section{Tiempo promedio de espera: Pte}

El tiempo promedio se asume como aquel en el que los pasajeros invierten desde su llegada hasta su salida de los sistemas de colas que se generan en cada parada de la ruta en análisis (Osais, 2018). Una de las medidas utilizadas para evaluar el rendimiento del sistema de colas de la ruta surge al formularse la siguiente pregunta: ¿Cuál es el tiempo promedio que los usuarios del servicio de transporte colectivo invierten en el sistema entero, incluyendo el tiempo de espera y de servicio? La Figura 6 del siguiente programa definido en la ventana processing responde la pregunta.

\section{Entidad}

Dos entidades conforman este modelo, una de ellas representa los pasajeros que se encuentran subiendo y bajando en las paradas, y la otra representa el autobús. Puesto que las entidades se mueven, hemos asignado una velocidad de 100 metros por minuto a los pasajeros que es aproximadamente la de una caminata normal; mientras que los autobuses se mueven con una velocidad promedio de 343.10 metros por minuto durante todo el trayecto de la ruta; línea 2 (Blanco \& Fajardo, 2001).

\section{Figura 6}

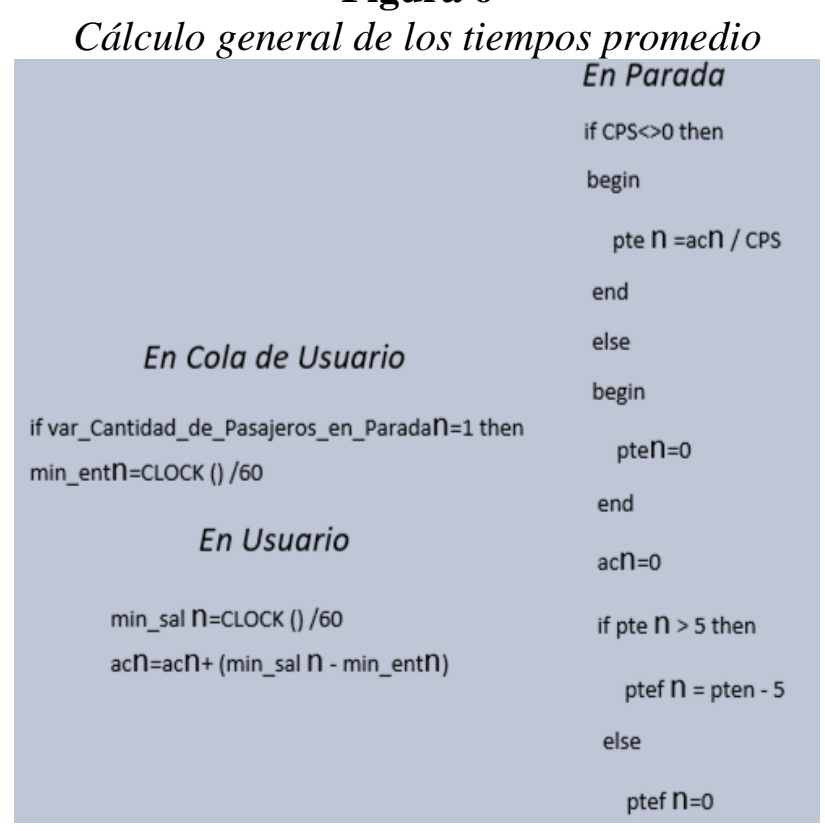

Nota: Línea de código que calcula los tiempos promedio de espera y de servicio, en cada n- ésima parada. Fuente: German Moreno 


\section{Atributo}

Un atributo es una característica común de todas las entidades (Kelton et al., 2008). Por ejemplo, la entidad bus tiene un atributo en el modelado que lo definimos como: attr_Capacidad_del_Bus $=\mathbf{4 0}$, también en el modelo se encuentran dos atributos más, a saber: attr_Cantidad_de_Pasajeros_en_el_Bus, y attr_Hora_de_entrada_al_Sistema

Variables de entrada y salida

La simulación de la mayoría de los sistemas de servicio es una simulación de terminación, porque tienen un evento de terminación obvio (es decir, hora de cierre) (Kyu \& Kang, 2013). La terminología, “operación de las paradas de bus” dice de la cantidad de pasajeros presentes en las paradas y de su tiempo de espera. En este contexto, las salidas numéricas son: 1. cantidad de pasajeros en la parada "CPP", y 2. Tiempo de espera "Pte". Cabe recordar que las dos variables mencionadas son las que constan como variables dependientes en la hipótesis de investigación.

Como salidas numéricas complementarias el modelo de simulación establece las siguientes: 1. El tiempo de espera forzado "Ptef" y 2. La cantidad de pasajeros desertores "CPD".

Con las salidas identificadas, el siguiente paso es identificar las variables de entradas. Éstas son valores numéricos que, una vez determinados, permiten iniciar la simulación y calcular todas las salidas requeridas. Estas entradas son: 1. Arribos del bus, 2. Arribos del pasajero a la parada, y 3 . Tiempos de servicio.

Los arribos del bus en el modelo son las frecuencias aleatorias con las que los vehículos ingresan al recorrido desde la Cooperativa de vivienda 24 de mayo. Estos tiempos obtenidos por medición directa se ajustan con una distribución uniforme: U $(120,480)$ segundos, la cual se programa en la ventana arrivals de ProModel, bajo la entidad bus.

El uso de un número semilla aleatorio diferente cambia el perfil del tráfico que llega y, por lo tanto, los resultados también pueden cambiar. Por lo tanto, se puede simular la variación estocástica de los tiempos de llegada del flujo de entrada (Macioszek \& Sierpiṅski, 2016).

\section{Cálculo del tamaño de muestra para estimar un valor de media}

Para comprender cuántas veces se debe repetir una simulación cuando el objetivo consiste en estimar la media de un valor de salida, es mejor empezar por escoger un número específico de corridas de simulación y preguntarse qué tan precisa es la estimación del 
valor de salida obtenido. Para responder a esta pregunta se requiere la creación de un intervalo de confianza alrededor de la estimación (Mathur \& Solow, 1996).

\section{Intervalo de confianza para la media verdadera}

Supongamos que se usa $n$ corridas de simulación para obtener un promedio estimado $\bar{X}$ para la media verdadera, $\mu$. La pregunta que nos planteamos ahora es: ¿qué tan cerca está este valor estimado $\bar{X}$ del valor verdadero de $\mu$ ?. Aplicando la teoría sobre estimación estadística, el objetivo es determinar un valor $e, \mathrm{y}$ crear un intervalo de la forma:

$$
\bar{X} \pm e \quad[1]
$$

de modo que, con un cierto grado de confianza, se pueda decir que la media verdadera, $\mu$, está en este intervalo. Seleccionamos el nivel de confianza en la forma de un valor de $\alpha$ entre cero y uno, de manera que con un nivel de confianza de $(1-\alpha) .100 \%$, podemos afirmar que el valor verdadero de $\mu$ está en el intervalo: $\bar{X} \pm e$ (Mathur \& Solow, 1996).

Por ejemplo, para obtener un nivel de confianza de 95\%, escogemos un valor de $\alpha=0.05$. Una vez elegido un valor apropiado de $\alpha$, calculamos el intervalo de confianza asociado mediante:

$$
\bar{X} \pm t_{(n-1), \alpha / 2} \cdot \frac{s}{\sqrt{n}}
$$

Donde:

$n$ : el tamaño de la muestra (es decir, el número de corridas de simulación)

$\bar{X}$ :promedio de muestra

$s$ : desviación estándar de la muestra

$t_{(n-1), \alpha / 2}$ : el valor de la distribución $t$ con $n-1$ grados de libertad

Notemos que en la expresión 3,

$$
e=t_{(n-1), \alpha / 2} \cdot \frac{s}{\sqrt{n}}
$$

En consecuencia, a este valor de e se le conoce como precisión de la estimación y representa una medida de qué tan fiable es la salida de la simulación. Para un valor fijo de $\alpha$, la precisión de la estimación puede mejorarse aumentando solamente el tamaño de la muestra, $n$, lo cual significa aumentar el número de réplicas o corridas de simulación. 
Cálculo del tamaño de muestra para lograr un nivel de confianza y una precisión dados

Ahora se verá cómo calcular el tamaño de la muestra $n$, para lograr una precisión deseada en la estimación. Supongamos que un cierto nivel de confianza $e_{1}$, calculado anteriormente y asociado con un intervalo de confianza digamos de $95 \%$, debe reducirse a un valor dado $e$. Este objetivo puede lograrse mediante el aumento del número de corridas de simulación de $n$ a un nuevo valor, $n^{*}$, tal que:

$$
\text { Precisión } e=t_{\left(n^{*}-1\right), \alpha / 2 .} \frac{s}{\sqrt{n^{*}}}
$$

Resolviendo para $n^{*}$ tenemos que:

$$
n^{*}=\left(\frac{\left.t^{*}{ }^{*}-1\right), \alpha / 2 . \mathrm{S}}{e}\right)^{2}
$$

Aunque se conoce el valor de $e$, el de $t_{\left(n^{*}-1\right), \alpha / 2}$ depende de $n^{*}$. Más aún, el valor de la desviación estándar de la muestra, $s$, no puede ser determinado hasta que se lleven a cabo las $n^{*}$ simulaciones (y todavía no se conoce el valor de $n^{*}$ ).

Para valores grandes de $n$, la distribución $t$ puede ser aproximada bastante bien por la distribución normal estándar, de modo que un planteamiento para resolver el problema de que $t_{\left(n^{*}-1\right), \alpha / 2}$ depende de $n^{*}$ consiste en sustituir $t_{\left(n^{*}-1\right), \alpha / 2}$ con el correspondiente valor de la normal estándar. Específicamente, $t_{\left(n^{*}-1\right), \alpha / 2}$ es sustituido por $Z_{\alpha / 2}$, de manera que el área bajo la curva de la distribución normal que se encuentra a la derecha de este valor es $\alpha / 2$. Esto es:

$$
n^{*}=\left(\frac{Z_{\alpha / 2 \cdot s}}{e}\right)^{2}
$$

Donde

$n^{*}:$ el número de corridas o réplicas de simulación.

Sin embargo, para resolver el hecho de que el valor de $s$ todavía no se conoce, se utiliza un enfoque alternativo, a saber:

1. Seleccionar un valor inicial para $n$

2. Efectuar $n$ corridas de simulación 
3. Sobre la base de los resultados de todas las corridas de simulación, calcular $\bar{x}, s$, luego determinar:

$$
n^{*}=\left(\frac{Z_{\alpha / 2} \cdot \mathrm{s}}{e}\right)^{2}
$$

4. Si $n \geq n^{*}$, detener el proceso de simulación. Pues, ya se han efectuado suficientes corridas de simulación. En cualquier otro caso, efectuar $n^{*}-n$ más corridas de simulación, antes de regresar al paso 3 (Mathur \& Solow, 1996).

\section{Resultados}

Los resultados que se obtienen giran en torno a los escenarios objeto de investigación y análisis que se encuentran en el objetivo general; así, para evaluar cómo influyen las demoras de los autobuses entre paradas, por dispositivos de control de tránsito, e indisciplinas de los conductores en el número de pasajeros y su tiempo de espera en las paradas de bus, determinamos tres escenarios de análisis; a saber: Dep+Dpdis+Dpi, Dep+Dpi y Dep.

El escenario general: Dep+Dpdis+Dpi, representa al sistema modelado en su totalidad, es decir, con las demoras entre paradas (Dep), las demoras por dispositivos de tránsito (Dpdis) y las demoras por indisciplinas de los conductores (Dpi). Los resultados simulados según este escenario, son importantes en virtud de la comparación que es posible realizar con los nuevos escenarios que se desprenden del modelo general. Las salidas numéricas obtenidas bajo simulación corresponden a las etiquetas: CPS, CPP, CPD, Pte, Tep y Ptef; donde: CPS, representa la cantidad de pasajeros servidos, CPP: cantidad de pasajeros en la parada, CPD: cantidad de pasajeros desertores, Pte: promedio de tiempo de espera, Tep: tiempo de espera planificado y Ptef: promedio de tiempo de espera forzado.

El modelo permite obtener resultados simulados si del escenario del sistema completo anulamos, por ejemplo, las demoras por indisciplinas (Dpi) para obtener un nuevo escenario: Dep+Dpdis. Ahora tenemos una nueva versión del modelo que con el soporte de ProModel nos permite observar las salidas numéricas bajo la influencia del par: Dep+Dpdis.

Si del escenario completo anulamos o retiramos todos los dispositivos para el control del tránsito, es decir, si suponemos que el sistema opera sin ningún dispositivo, obtenemos un nuevo modelo del tipo: Dep+Dpi. Ahora, la simulación permite observar salidas numéricas bajo la influencia de las demoras entre paradas e indisciplinas de los conductores. El objetivo es estimar y observar el efecto que bajo este tratamiento experimental se produce en el número de pasajeros y su tiempo de espera. 
Por último, si del modelo completo retiramos las demoras por dispositivos y las provocadas por indisciplinas de los conductores, entonces estamos en capacidad de observar el comportamiento de las paradas de autobús bajo la influencia de las demoras entre paradas (Dep). Es evidente que crear un escenario sin las demoras producidas entre paradas es imposible.

Variable dependiente: número de pasajeros

Los promedios de pasajeros por bus, en todo el sistema de ruta de la línea 2, por cada grupo de demoras contempladas en la hipótesis de investigación se muestra en la tabla 1. No obstante, el primer grupo "Dep+Dpdis+Dpi" de las primeras columnas de las tablas 1 y 2 , presentan los promedios de pasajeros y tiempos de espera, respectivamente, en todo el sistema en condiciones normales. Sin embargo, de no emplearse en la prueba de hipótesis los registramos para compararlos con los que sí intervienen en la misma.

Tabla 1

Promedios de pasajeros por bus en todo el sistema

\begin{tabular}{ccccc}
\hline Bus & Dep+Dpdis+Dpi & Dep+Dpdis & Dep+Dpi & Dep \\
\hline 1 & 2.310 & 2.380 & 2.314 & 2.166 \\
\hline 2 & 1.490 & 1.560 & 1.522 & 1.534 \\
\hline 3 & 1.251 & 1.283 & 1.231 & 1.272 \\
\hline 4 & 1.152 & 1.173 & 1.129 & 1.142 \\
\hline 5 & 1.129 & 1.139 & 1.084 & 1.089 \\
\hline 6 & 1.091 & 1.059 & 1.130 & 1.126 \\
\hline 7 & 1.127 & 1.092 & 1.106 & 1.085 \\
\hline 8 & 1.128 & 1.129 & 1.125 & 1.123 \\
\hline 9 & 1.159 & 1.107 & 1.125 & 1.129 \\
\hline 10 & 1.167 & 1.093 & 1.124 & 1.110 \\
\hline 11 & 1.114 & 1.133 & 1.136 & 1.058
\end{tabular}

Fuente: German Moreno

Variable dependiente: tiempo de espera

Los tiempos promedios de tiempo de espera de los pasajeros, por bus, en todo el sistema de ruta de la línea 2, por cada grupo de demoras contempladas en la hipótesis de investigación se muestran en la tabla 2.

\section{Tabla 2}

Promedios de tiempos de espera por bus en todo el sistema

\begin{tabular}{ccccc}
\hline Bus & $\begin{array}{r}\text { Dep+Dpdis+Dpi } \\
(\min )\end{array}$ & Dep+Dpdis (min) & Dep+Dpi (min) & Dep (min) \\
\hline 1 & 1.489 & 1.392 & 1.404 & 1.532 \\
\hline 2 & 1.796 & 1.760 & 1.820 & 1.960 \\
\hline 3 & 2.044 & 1.941 & 2.002 & 2.129 \\
\hline
\end{tabular}


Tabla 3

Promedios de tiempos de espera por bus en todo el sistema (continuación) $\begin{array}{llll}\text { Bus } & \text { Dep+Dpdis+Dpi } & \text { Dep+Dpdis (min) } & \text { Dep+Dpi (min) }\end{array}$ (min)

\begin{tabular}{ccccc}
\hline 4 & 2.272 & 1.997 & 2.121 & 2.164 \\
\hline 5 & 2.430 & 2.254 & 2.395 & 2.383 \\
\hline 6 & 2.476 & 2.318 & 2.440 & 2.509 \\
\hline 7 & 2.517 & 2.338 & 2.387 & 2.508 \\
\hline 8 & 2.511 & 2.406 & 2.480 & 2.576 \\
\hline 9 & 2.663 & 2.444 & 2.431 & 2.445 \\
\hline 10 & 2.523 & 2.432 & 2.375 & 2.440 \\
\hline 11 & 2.418 & 2.384 & 2.355 & 2.451 \\
\hline 12 & 2.366 & 2.495 & 2.208 & 2.432 \\
\hline
\end{tabular}

Fuente: German Moreno

\section{Discusión}

Los resultados simulados de la tabla 1, evidencian para los cuatro escenarios un asombroso grado de regularidad de pasajeros durante el recorrido de los doce buses. Los promedios de pasajeros obtenidos de todas las 51 paradas oficiales por cada bus en condiciones normales del sistema y en función de su correspondiente número de réplicas, reflejan el comportamiento de las mismas a medida que transcurre el tiempo. Esto se observa en la columna: Dep+Dpdis+Dpi, de la tabla 1 y la figura 7.

\section{Figura 7}

Gráfico de línea que muestra la demanda de clientes de 6 h30 a 9 h30

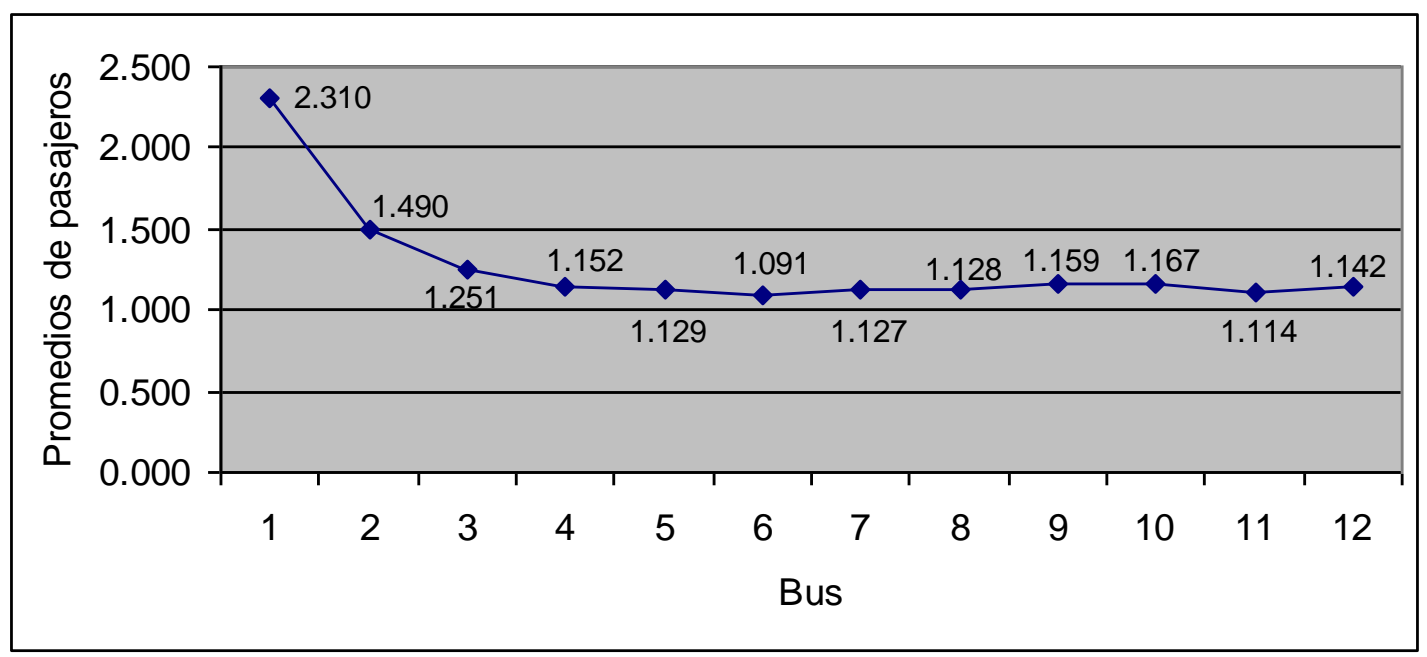

Nota: Distribución de los pasajeros por bus según el orden de salida a partir de las 6h30. Fuente: German Moreno.

Los valores promedios de pasajeros obtenidos del sistema en condiciones normales (Ver columna: Dep+Dpdis+Dpi, de la tabla 1 y figura 7) nos muestran que hasta el recorrido 
del sexto bus existe un decrecimiento de promedios progresivo leve. Por el contrario, a partir del recorrido del séptimo bus, los valores crecen ligeramente hasta cuando el décimo bus completa su recorrido.

\section{Figura 8}

Gráfico de línea que muestra los "Pte" de clientes de 6 h30 a 9 h30

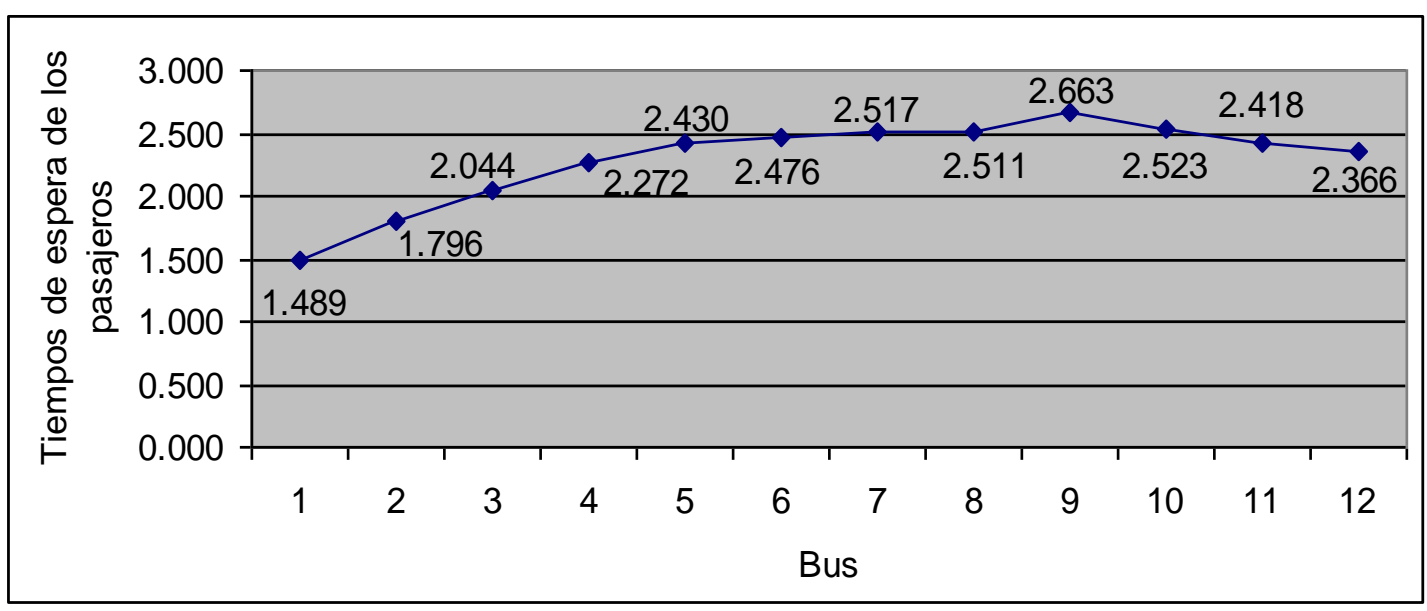

Nota: Distribución de los tiempos de espera por bus en el orden de salida a partir de las 6 h30.

Fuente: German Moreno

El análisis de datos (ver columna: Dep+Dpdis+Dpi, de la tabla 2) de los tiempos promedios de espera que experimentan los usuarios de la línea 2 , en condiciones normales, muestra que, para los doce buses, las esperas en las paradas del sistema no exceden, peor se acercan a los cinco minutos de tiempo de espera planificado por parte de los pasajeros. Este hecho se comprueba en el gráfico de la Figura 8, donde se observan los tiempos promedios de espera por cada bus, a partir de las $6 \mathrm{~h} 30$.

\section{Conclusiones}

- Las pruebas de simulación ejecutadas y visualizadas en sus variables cuantitativas de salida con el apoyo de herramientas de gráficos de la hoja de cálculo de Excel, evidencian que no hay largos tiempos de espera; como tampoco, existe aglomeración en las paradas, debido a los distintos tipos de demoras que experimentan los autobuses. Lo dicho, se puede corroborar con la realidad; es decir, los largos tiempos de espera y acumulación de usuarios en las paradas se descartan en virtud de la presencia de otras líneas de buses que convergen en una gran parte de la ruta modelada.

- Por otro lado, está el comportamiento probabilístico del sistema, el cual, permite concluir que los valores promedios del número de pasajeros en las paradas guardan estrecha relación con los horarios de mayor demanda que se presentan en el periodo de 6 h30 a 9 h30. 
- Al observar las salidas numéricas-por parada de bus- de las variables cantidad de pasajeros "CPP" y cantidad de pasajeros servidos "CPS" concluimos que en la mayoría de paradas de bus, el número de pasajeros que esperan el vehículo, son el mismo número que reciben servicio. La experiencia confirma que esto es así. En general, los resultados muestran que las quejas de los usuarios más bien pueden ser de tipo esporádicas, debido a interrupciones no periódicas que el servicio de buses pueden experimentar.

\section{Referencias Bibliográficas}

Barceló, J. (2010). Fundamentals of Traffic Simulation. In J. Barceló. Springer.

Blanco Rivero, L. E., \& Fajardo Piedrahita, I. D. (2001). Simulación con PROMODEL. In Casos de producción y logística. Escuela Colombiana de Ingenieria.

Cárdenas, J. (2018). Ingeniería de Tránsito Fundamentos y Aplicaciones (Novena edición ed.). México: Alfaomega Grupo Editor, S.A.

García, E., García Reyes, H., \& Cardenás, L. (2013). Simulación y Análisis de Sistemas con ProModel. Monterrey, México: Pearson.

International Journal os Scientific Research in Science and Technology. (2018). Technoscience Academy.

Kelton, D. W., Sadowski, R. P., \& Sturrock, D. T. (2008). Simulación con software Arena. México: McGrawHill.

Kobayashi, H., Mark, B., \& Turin, W. (2012). Probability. Cambridge.

Kyu Choi, B., \& Kang, D. (2013). Modeling and Simulation od Discrete - Event Systems. New Jersey: Wiley.

Levine , D., Krehbiel, T., \& Berenson, M. (2006). Estadística para administración. México: Pearson.

Macioszek, E., \& Sierpinski, G. (2016). Comtemporary Challenges of Transport Systems and Traffic Engineering. Poland.

Mathur, K., \& Solow, D. (1996). El arte de la toma de decisiones. México: Pearson Educación.

Ortúzar, J. (2015). Modelos de Demanda de Transporte. In J. d. Ortúzar. Chile: Alfaomega. 
Osais, Y. (2018). Computer Simulation A Foundation Approach Using Python. CRC Press.

Pazos Arias, J. J., Suaréz González, A., \& Díaz Redondo, R. (2003). Teoría de Colas y Simulación de Eventos Discretos. Madrid: Pearson Educación S.A.

Walpole, R., Myers, R., Myers, S., \& Ye, K. (2012). Probabilidad y estadística para ingeniería y ciencias. México: Pearson.

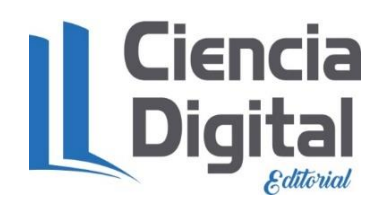


El artículo que se publica es de exclusiva responsabilidad de los autores y no necesariamente reflejan el pensamiento de la Revista Alfa Publicaciones.

\section{Ciencia}

El artículo queda en propiedad de la revista y, por tanto, su publicación parcial y/o total en otro medio tiene que ser autorizado por el director de la Revista Alfa Publicaciones.
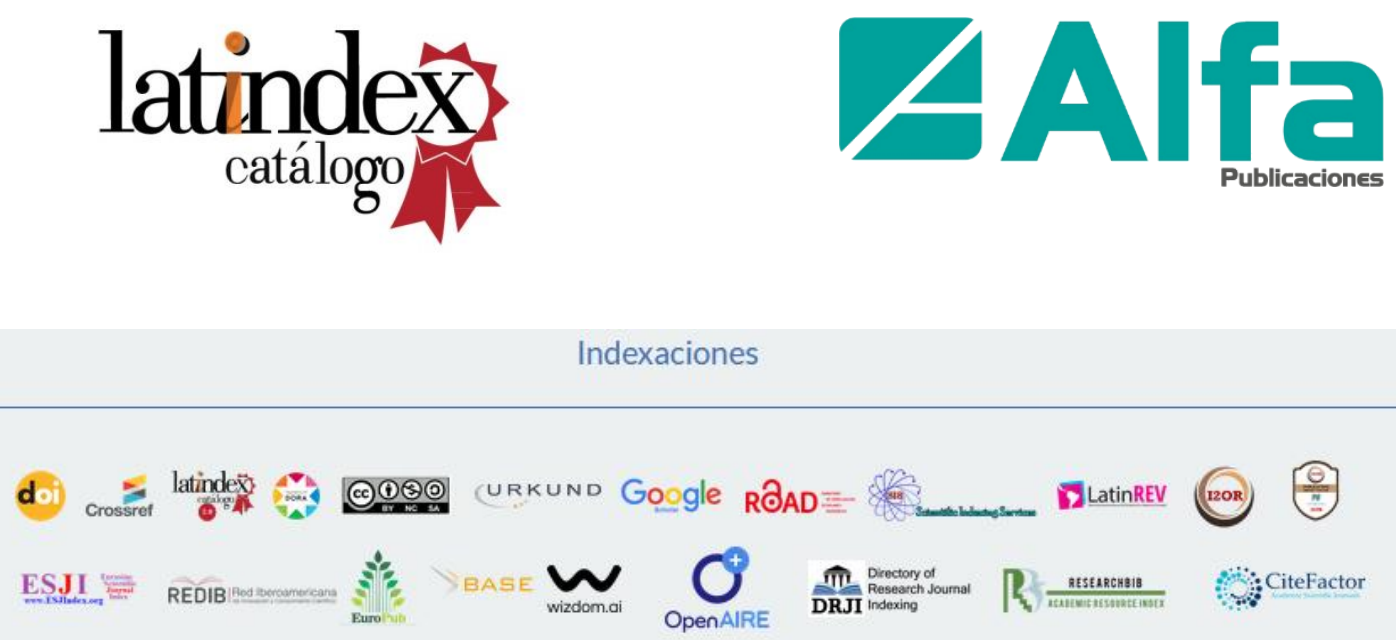